\title{
AN ALGEBRA OF FUNCTIONS
}

\author{
BJÖRN E. J. DAHLBERG
}

\begin{abstract}
It is established that the space of logarithmic potentials of $H^{1}$-functions is closed under multiplication.
\end{abstract}

In this note we shall establish that the product of two functions, both of which are logarithmic potentials of $H^{1}$-functions, is the logarithmic potential of a function in $H^{1}$. Here $H^{1}=H^{1}\left(R^{n}\right)$ is defined as the class of those $L^{1}$-functions $f$ on $R^{n}$ for which it is possible to find $L^{1}$-functions $g_{1}, \ldots, g_{n}$ such that $\hat{g}_{j}(\xi)=\xi_{j}|\xi|^{-1} \hat{f}(\xi)$, where $\hat{f}$ denotes the Fourier transform of $f$. The $H^{1}$-norm of $f$ is then

$$
\|f\|=\|f\|_{1}+\sum_{1}^{n}\left\|g_{j}\right\|_{1} .
$$

For properties of $H^{1}$ see Stein [5] and Fefferman and Stein [3].

We define the logarithmic potential of a function $f$ as

$$
K f(x)=\int_{R^{n}} f(y) \log |x-y| d y .
$$

We can now state our main result.

TheOrem. If $f, g \in H^{1}$ then there is a function $h \in H^{1}$ such that $K f K g=$ $K h$. Moreover, there is a constant $C$ independent of $f$ and $g$ such that $\|h\| \leqslant$ $C\|f\|\|g\|$.

We remark that in the case $n=1$ this type of algebra has been studied by Coifman and Weiss [2, p. 601] in connection with the algebra of CalderonZygmund operators on $R^{2}$ with characteristic in $H^{1}(T)$.

For other results when spaces of potentials are closed under multiplication see Strichartz [6], [7], Bagby [1].

The proof of Theorem 1 is based on the atomic decomposition of $H^{1}$ functions. We recall that a function $a \in L^{\infty}\left(R^{n}\right)$ is called an atom if there is a cube $I$ with its sides parallel to the coordinate axis such that $a$ is supported on $I, \int_{R^{n}}$ a $d x=0$, and $\|a\|_{\infty} \leqslant|I|^{-1}$. Here $|E|$ denotes the Lebesgue measure of a set $E \subset R^{n}$.

It is now known that given $f \in H^{1}$ there is a sequence of atoms $a_{j}$ and

Received by the editors April 21, 1978.

AMS (MOS) subject classifications (1970). Primary $31 \mathrm{~B} 99$.

Key words and phrases. Logarithmic potentials, $H^{1}$-functions, atoms, Sobolev inequalities. 
numbers $\lambda_{j}$ such that

$$
f=\sum \lambda_{j} a_{j}
$$

and $\Sigma\left|\lambda_{j}\right| \leqslant C\|f\|$ for some fixed constant $C$. Also, whenever the decomposition (1) holds $\|f\| \leqslant C \Sigma\left|\lambda_{j}\right|$ for some fixed constant $C$. For a full discussion of these results see Coifman and Weiss [2]. From these results it follows that in order to prove the theorem it is sufficient to show that if $a$ and $b$ are two atoms then $K a K b=K h$ for some $h \in H^{1}$ such that $\|h\| \leqslant C$, where $C$ only depends on $n$.

We recall that a function $\phi$ is said to have bounded mean oscillation if for the family of cubes $I$ we have

$$
\sup |I|^{-1} \int_{I}\left|\phi-\phi_{I}\right| d x=\|\phi\|_{*}<\infty,
$$

where $\phi_{I}=|I|^{-1} \int_{I} \phi d x$. The space of all such functions is denoted by BMO. An important fact is that the dual of $H^{1}$ is BMO; see Fefferman and Stein [3]. We shall need the following estimate, whose proof is a straightforward modification of inequality (1.2) in Fefferman and Stein [3]. If $\phi \in$ BMO and $1 \leqslant p<\infty$ then for any cube $I$ centered at 0 we have

$$
\left(\int \alpha(\alpha+|x|)^{-1-n}\left|\phi(x)-\phi_{I}\right|^{P} d x\right)^{1 / P} \leqslant C\|\phi\|_{*},
$$

where $\alpha$ is the side of $I$ and $C$ is independent of $\phi$.

For a cube $I$ we denote by $2 I$ the cube with the same center as $I$ but with twice the side. With the support cube of an atom $a$ we mean the smallest (axis parallel) cube which contains the support of $a$.

Lemma. Suppose $a$ is an atom, the support cube of which is centered at 0. There is a constant $C$ only depending on $n$ such that if $x \notin 2 I$ and $0 \leqslant j \leqslant n$ then

$$
\left|\nabla_{j} K a(x)\right| \leqslant C \alpha(|x|+\alpha)^{-1-j} .
$$

If $x \in 2 I$ and $0 \leqslant j \leqslant n-1$ then

$$
\left|\nabla_{j} K a(x)\right| \leqslant C \alpha^{-j}
$$

Here $\alpha$ is the side of $I$ and $\nabla_{j} h$ denotes the partial derivatives of $h$ of order $j$ arranged in some order.

Proof. Let $F_{j}(x)=\nabla_{j} \log |x|$. Since $\left|F_{j}(x)\right| \leqslant C_{j}|x|^{-j}, 1 \leqslant j$, it follows that if $x \notin 2 I$, then

$$
\begin{aligned}
\nabla_{j} K a(x) & =\int_{I} a(y) F_{j}(x-y) d y \\
& =\int_{I} a(y)\left(F_{j}(x-y)-F_{j}(x)\right) d y .
\end{aligned}
$$

Hence $\left|a_{j} K a(x)\right| \leqslant C_{j}|x|^{-j-1}$ which shows the first part of the lemma. Since 
$\|a\| \leqslant C$ and $\log |x| \in$ BMO (see John and Nirenberg [4]) it follows from the duality between $H^{1}$ and BMO that

$$
\|K a\|_{\infty} \leqslant C \text {. }
$$

If $1 \leqslant j \leqslant n-1$ and $x \in 2 I$ then

$$
\begin{aligned}
\left|\nabla_{j} K a(x)\right| & \leqslant \int\left|F_{j}(x-y)\right||a(y)| d y \\
& \leqslant C_{j} \int_{|x| \leqslant \alpha}|y|^{-j}|I|^{-1} d y \leqslant C_{j} \alpha^{-j},
\end{aligned}
$$

which completes the proof of the lemma.

We shall now assume that $a$ and $b$ are atoms with support cubes $I$ and $J$ respectively. We shall assume that $a$ and $b$ are $C^{\infty}$ and we shall next show that there is a constant $C$, only depending on $n$, such that for all $\phi \in$ BMO we have

$$
\left|\int_{R^{n}} \phi D^{n}(K a K b) d x\right| \leqslant C\|\phi\|_{*} .
$$

Here $D$ denotes differentiation in the direction of a unit vector $e$.

We let $\alpha$ and $\beta$ denote the sides of $I$ and $J$ respectively. It is no loss in generality in assuming $\alpha \leqslant \beta$ and $I$ is centered at the origin. Fix $j, 0 \leqslant j \leqslant$ $n$, and put

$$
A_{j}=\int_{R^{n}-2 I}\left(\phi-\phi_{2 I}\right) D^{j} K a D^{n-j} K b
$$

Defining $p=p_{j}=(n+1)(j+1)^{-1}$ we have

$$
1-p^{-1}=n\left(p^{-1}-k n^{-1}\right) \text {. }
$$

From the lemma, (2) and Hölder's inequality we get

$$
\begin{aligned}
\left|A_{j}\right| & \leqslant C \int_{R^{n}-2 I} \alpha(|x|+\alpha)^{-j-1}\left|\phi(x)-\phi_{2 I}\right|\left|D^{n-j} K b(x)\right| d x \\
& \leqslant C \alpha^{1-1 / p}\|\phi\|_{*}\left\|D^{n-j} K b\right\|_{q},
\end{aligned}
$$

where $q=p / p-1$. If $j=0$ then $1<q<\infty$ and since

$$
\widehat{D^{n} K b}(\xi)=\langle e, \xi\rangle^{n}|\xi|^{-n} \hat{b}(\xi)
$$

it follows from well-known results on multipliers (see Stein [5]) that $\left\|D^{n} I b\right\|_{q}$ $\leqslant C\|b\|_{q} \leqslant C \beta^{-n\left(1-q^{-1}\right)}$. Here $\langle e, \xi\rangle$ denotes the scalar product. Remembering (5) and the assumption $\alpha \leqslant \beta$ we see that $\left|A_{0}\right| \leqslant C$. If $1 \leqslant j \leqslant n-1$ we observe that

$$
\left|D^{n-j} K b\right| \leqslant C I_{j}|b|
$$

where $I_{j} f(x)=\int f(y)|x-y|^{j-n} d y$. It is known (see Stein [5, Chapter V] that if $r$ is defined by $q^{-1}=r^{-1}-j n^{-1}$ then $\left\|I_{j} f\right\|_{q} \leqslant C\|f\|_{r}$. Hence $\left\|D^{n-j} K b\right\|_{q}$ $\leqslant C \beta^{-n\left(1-r^{-1}\right)}$. From (5) and the assumption $\alpha \leqslant \beta$ it follows that $\left|A_{j}\right| \leqslant C$. 
If $j=n$ it follows from (3) that $\left|A_{n}\right| \leqslant C\|\phi\|_{*}$ so we have in all cases

$$
\left|A_{j}\right| \leqslant C\|\phi\|_{*}, \quad 0 \leqslant j \leqslant n .
$$

We shall now put

$$
B_{j}=\int_{2 I}\left(\phi-\phi_{2 I}\right) D^{j} K a D^{n-j} K b d x
$$

If $1 \leqslant j \leqslant n-1$ it follows from the lemma that $\left|D^{j} K a D^{n-j} K b\right| \leqslant C \alpha^{-j} \beta^{j-n}$ $\leqslant C|I|^{-1}$. Hence

$$
\left|B_{j}\right| \leqslant C|I|^{-1} \int_{2 I}\left|\phi-\phi_{2 I}\right| d x \leqslant C\|\phi\|_{*}
$$

From (3) it follows that

$$
\left|B_{0}\right|+\left|B_{n}\right| \leqslant C\left(\int_{2 I}\left|\phi-\phi_{2 I}\right|^{2} d x\right)^{1 / 2}\left(\left\|D^{n} K b\right\|_{2}+\left\|D^{n} K a\right\|_{2}\right) .
$$

It is known that $\left(|I|^{-1} \int_{I}\left|\phi-\phi_{I}\right|^{2}\right)^{1 / 2} \leqslant C\|\phi\|_{*}$, see John and Nirenberg [4]. Since

$$
\begin{aligned}
\left\|D^{n} K a\right\|_{2}+\left\|D^{n} K b\right\|_{2} & \leqslant C\left(\|a\|_{2}+\|b\|_{2}\right) \\
& \leqslant C\left(|I|^{-1 / 2}+|J|^{-1 / 2}\right) \leqslant C|I|^{-1 / 2}
\end{aligned}
$$

it follows that

$$
\left|B_{j}\right| \leqslant C\|\Phi\|_{*}, \quad 0 \leqslant j \leqslant n .
$$

Since $\int_{R^{n}} D^{n}(K a K b) d x=0$ we have

$$
\int_{R^{n}} \phi D^{n}(K a K b) d x=\sum_{0}^{n}\left(\begin{array}{l}
n \\
j
\end{array}\right)\left(A_{j}+B_{j}\right)
$$

which yields (4).

To prove the theorem it is now sufficient to show that if $F=K a K b$ then there is a function $h \in H^{1}$ such that $\hat{h}(\xi)=|\xi|^{n} \hat{F}(\xi)$ and $\|h\| \leqslant C$.

Let $f_{i}, 1 \leqslant i \leqslant n$, be defined by $\hat{f}_{i}(\xi)=\xi_{i}^{n} \hat{F}(\xi)$. From (4) follows $\left\|f_{i}\right\| \leqslant C$. If $T_{i}$ denotes the operator defined by $\widehat{T_{i}(f)}(\xi)=\xi_{i}^{n}|\xi|^{-n} \hat{f}(\xi)$ it follows from theory of multipliers on $H^{1}$ (see Stein [5, Chapter VII]) that $\left\|T_{i} f\right\| \leqslant C\|f\|$. Hence $\|g\| \leqslant C$, where $g=\sum_{1}^{n} T_{i} f_{i}$. If $T$ denotes the operator defined by $\widehat{T f}(\xi)=|\xi|^{2 n}\left(\sum_{1}^{n} \xi_{i}^{2 n}\right)^{-1} \hat{f}(\xi)$, then $T$ is bounded on $H^{1}$. Finally, observing that $\widehat{T g}(\xi)=|\xi|^{n} \hat{F}(\xi)$ yields the theorem.

\section{REFERENCES}

1. R. Bagby, Lebesgue spaces of parabolic potentials, Illinois J. Math. 15 (1971), 610-635.

2. R. R. Coifman and G. Weiss, Extensions of Hardy spaces and their use in analysis, Bull. Amer. Math. Soc. 83 (1977), 569-645.

3. C. Fefferman and E. M. Stein, $H^{p}$ spaces of several variables, Acta Math. 129 (1972), 137-193.

4. F. John and L. Nirenberg, On functions of bounded mean oscillation, Comm. Pure Appl. Math. 14 (1961), 415-426. 
5. E. M. Stein, Singular integrals and differentiability properties of functions, Princeton Math. Series, No. 30, Princeton Univ. Press, Princeton, N. J., 1970.

6. R. S. Strichartz, Multipliers on fractional Sobolev spaces, J. Math. Mech. 16 (1967), 1031-1060.

7. __ A note on Sobolev algebras, Proc. Amer. Math. Soc. 29 (1971), 205-207.

Department' OF Mathematics, UNIVERsity of Göteborg and Chalmers UNIVERsity of TeChNology, FACK, S-40220 GöTEBORg, SWEdEN 